\title{
Noninvasive photoacoustic sentinel lymph node mapping using Au nanocages as a lymph node tracer in a rat model
}

Kwang Hyun Song, Chulhong Kim, Claire M. Cobley, Younan Xia, Lihong V. Wang

Kwang Hyun Song, Chulhong Kim, Claire M. Cobley, Younan Xia, Lihong V. Wang, "Noninvasive photoacoustic sentinel lymph node mapping using Au nanocages as a lymph node tracer in a rat model," Proc. SPIE 7177, Photons Plus Ultrasound: Imaging and Sensing 2009, 71772L (24 February 2009); doi: $10.1117 / 12.809676$

SPIE. Event: SPIE BiOS, 2009, San Jose, California, United States 


\title{
Noninvasive photoacoustic sentinel lymph node mapping using Au nanocages as a lymph node tracer in a rat model
}

\author{
Kwang Hyun Song, Chulhong Kim, Claire M. Cobley, Younan Xia, and Lihong V. Wang \\ Department of Biomedical Engineering, Washington University in St. Louis, \\ Campus Box 1097, One Brookings Drive, St. Louis, Missouri 63130-4899
}

\begin{abstract}
Sentinel lymph node biopsy (SLNB) has been widely performed and become the standard procedure for axillary staging in breast cancer patients. In current SLNB, identification of SLNs is prerequisite, and blue dye and/or radioactive colloids are clinically used for mapping. However, these methods are still intraoperative, and especially radioactive colloids based method is ionizing. As a result, SLNB is generally associated with ill side effects. In this study, we have proposed near-infrared Au nanocages as a new tracer for noninvasive and nonionizing photoacoustic (PA) SLN mapping in a rat model as a step toward clinical applications. Au nanocages have great features: biocompatibility, easy surface modification for biomarker, a tunable surface plasmon resonance (SPR) which allows for peak absorption to be optimized for the laser being used, and capsule-type drug delivery. Au nanocage-enhanced photoacoustic imaging has the potential to be adjunctive to current invasive SLNB for preoperative axillary staging in breast cancer patients.

Keywords: photoacoustic, nanocages, sentinel lymph node, mapping,
\end{abstract}

\section{INTRODUCTION}

Various shape and material of nanoparticles have been widely used for a number of biological and medical applications since they have been discovered can be utilized efficiently for delivering $(1,2)$ and $\operatorname{targeting}(3,4)$ therapeutic agents to a specific site. In addition to therapeutic procedures, nanoparticles are widely used for diagnostic purposes, $(3,5,6)$ as they can be engineered to enhance the contrasts of imaging modalities such as optical imaging, $(3,7$, 8) magnetic resonance imaging (MRI),(9) and computed tomography (CT).(10) Nanoparticles have widely tunable properties such as sizes, shapes, and compositions, so their application in imaging should be broad. In diagnosis, nanomaterials can be used to identify sentinel lymph nodes (SLNs) for axillary staging in breast cancer patients.

Sentinel lymph node biopsy (SLNB) is widely performed and has become the standard for axillary staging. In SLN mapping as a prerequisite for SLNB, a blue dye (e.g., isosulfan blue, or methylene blue) and/or nanometer-sized radioactive colloids (e.g., technetium-99 $\left.\left({ }^{99 \mathrm{~m}} \mathrm{Tc}\right)\right)$ are usually used in the clinic. $(11,12)$ However, these methods have several limitations: 1) The method using a blue dye requires a surgical operation to expose a SLN for visual detection and also necessitates some experienced hands. 2) This method often leaves a blue stain on the injection point in the skin for months after the surgery.(13) 3) The radioactive colloid method not only requires special facilities for handling radioactive isotope materials but also suffers from the low spatial resolution of Geiger counters for noninvasive SLN identification.(14) In addition, the method takes about 24 hours for nodal uptake due to the large size of the tracers (typically 100-200 nm) and may cause radiation damage to the connective tissues of the skin. Therefore, noninvasive, nonionizing, and high-resolution mapping of SLNs would be beneficial to breast cancer patients, and would greatly aid minimally invasive staging methods such as fine needle aspiration biopsy (FNAB).(15)

MRI has been tried with an ultra-small superparamagnetic iron oxide (USPIO, 17-20 $\mu \mathrm{m}(16)$ ) contrast agent for SLN mapping. $(17,18)$ Even though MRI provides an excellent imaging depth and spatial resolution, it still suffers from low sensitivity and high cost. Furthermore, patients need to be transported from an operating room to a MRI unit since most operating rooms do not have MRI capabilities. On the other hand, optical imaging has several orders of magnitude higher sensitivity than MRI, and can be readily and easily adapted to the clinic.(14) Optical imaging is also nonionizing and relatively inexpensive. Therefore, optical lymph node tracers, such as fluorescent beads(14) (20-200 nm) and quantum dots $(7,19)(15-20 \mathrm{~nm})$, have been studied for noninvasive SLN mapping based on fluorescent imaging. However, these methods have poor spatial resolution at the regions beyond one transport mean free path $(\sim 1 \mathrm{~mm})$, resulting in inaccurate localization of SLNs since the average depth of SLNs is $12 \pm 5 \mathrm{~mm}$ in human (from the top surface

Photons Plus Ultrasound: Imaging and Sensing 2009, edited by Alexander A. Oraevsky, Lihong V. Wang, Proc. of SPIE Vol. $7177,71772 \mathrm{~L} \cdot$ C 2009 SPIE · CCC code: 1605-7422/09/\$18 · doi: $10.1117 / 12.809676$ 
of SLNs to the skin surface).(20) Moreover, the methods using quantum dots can create potential heavy metal toxicity in clinical use.(21)

Recently, Wang group demonstrated PA SLN mapping using clinically available methylene blue.(22) PA imaging modality is an emerging hybrid technology which is highly sensitive to endogenous and exogenous optical contrasts and provides good ultrasonic spatial resolution. Since this imaging technique uses diffusive photons, it can achieve upto $\sim 50$ mm imaging depth in biological tissues.(23) PA imaging for SLN mapping demonstrated a depth capability of $\sim 31 \mathrm{~mm}$ with satisfactory spatial resolution and clinical potential. However, methylene blue based PA mapping poses potential challenges. For example, since the methylene blue molecule $(0.7 \mathrm{~nm}$ in diameter and $1.6 \mathrm{~nm}$ in length) is smaller than 5 $\mathrm{nm}$ and easily flown into the echelon lymph nodes, there is a high possibility of false positives. ${ }^{(18,24)}$ Methylene blue molecule is also difficult to be bioconjugated to form target molecular probes. Since hemoglobin at the peak absorption wavelength of methylene blue $(677 \mathrm{~nm}$, PA excitation: $655 \mathrm{~nm})$ has stronger optical absorption than in the near-infrared (NIR) regions, it is hard to achieve relatively deep penetration depth.

In this study, for the first time to our knowledge, we apply NIR Au nanocages as a lymph node identifier to a noninvasive PA imaging modality. Au nanocages have attractive features: biocompatibility, easy surface modification for selective targeting, $(25,26)$ no potential heavy metal toxicity, $(21)$ a broad range of sizes $(35-100 \mathrm{~nm})$, tuning of the localized surface plasmon resonance (LSPR) peak, strong optical absorption in the NIR region, $(26,27)$ and encapsulated site-specific drug delivery.(28) In a rat model, we have successfully identified an in vivo SLN with enhanced contrast by $\mathrm{Au}$ nanocages and good spatial resolution noninvasively. In addition, we have demonstrated the depth capability of Au nanocages in SLN mapping as deep as $\sim 33 \mathrm{~mm}$. We expect that noninvasive SLN mapping based on Au nanocages can be beneficial to breast cancer patients. Going forward, by employing surface-modified Au nanocages, which can bind to specific breast cancer cells, this technique may constitute a noninvasive means of SLNB

\section{SYSTEM DESIGN AND METHOD}

Au nanocages. Au nanocages are cubic nanoboxes with seamless hollow interiors and porous walls (inset in Figure 1). They can be conveniently synthesized through a simple galvanic replacement reaction between $\mathrm{Ag}$ nanocubes and chloroauric acid $\left(\mathrm{HAuCl}_{4}\right)$ in water:(28, 29)

$$
3 \mathrm{Ag}(\mathrm{s})+\mathrm{AuCl}_{4}^{-}(\mathrm{ag}) \rightarrow \mathrm{Au}(\mathrm{s})+3 \mathrm{Ag}^{+}(\mathrm{ag})+4 \mathrm{Cl}^{-}(\mathrm{aq})
$$

The Ag nanocubes were, in turn, prepared using a rapid, sulfide-mediated polyol process with poly(vinyl pyrrolidone) (PVP) serving as a capping agent. The LSPR peak of the Au nanocages was tuned to $735 \mathrm{~nm}$ by controlling the ratio between $\mathrm{Ag}$ and $\mathrm{HAuCl}_{4}$ (Figure 1). An average edge length of the Au nanocages used for this study was $50.3 \pm 6.7 \mathrm{~nm}$ (standard deviation). The concentration of Au nanocages for the imaging study was $2 \mathrm{nM}$ in water. The Au nanocages were stabilized with PVP.

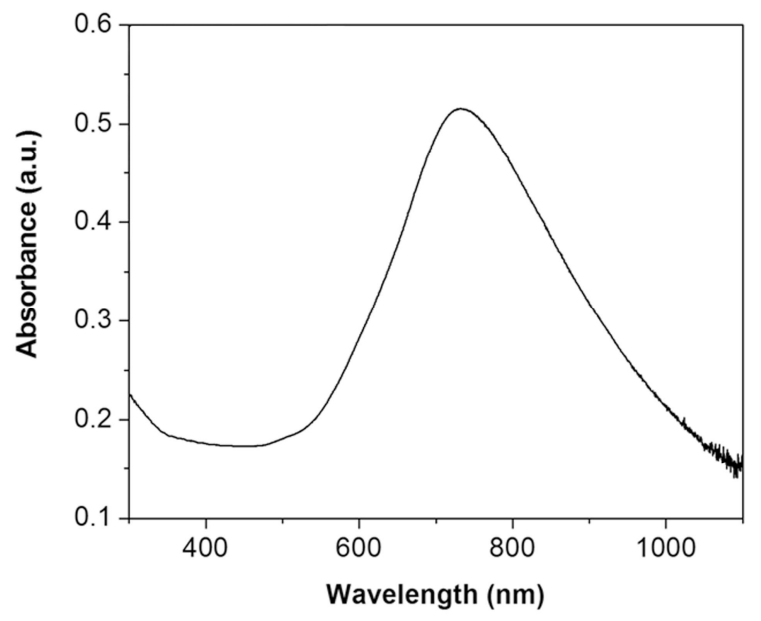

Figure 1. Absorption spectrum of Au nanocages. Wavelength at peak optical absorption was $\sim 735 \mathrm{~nm}$. The average edge length was $50.3 \pm 6.7 \mathrm{~nm}$ (standard deviation). The Au nanocages were stabilized with polyvinyl pyrrolidone (PVP). 
Imaging system. A reflection-mode PA imaging system (Figure 2) was employed for identifying SLNs following Au nanocage injection (see ref.(22) for a detailed description of the system). For PA excitation, a pulsed laser system with $755 \mathrm{~nm}$ wavelength was employed. This wavelength was close to the optical absorption peak of the Au nanocages. The laser light delivered to the skin was sufficiently broadened to conform to the maximum permissible exposure (MPE) limitation (actual fluence: $11 \mathrm{~mJ} / \mathrm{cm}^{2}$, MPE: $26 \mathrm{~mJ} / \mathrm{cm}^{2}$ ).(30) To detect PA waves with low acoustic attenuation, a 3.5 $\mathrm{MHz}$ or a $5 \mathrm{MHz}$ central frequency ultrasonic transducer (V380, V308, Panametrics-NDT) was used.

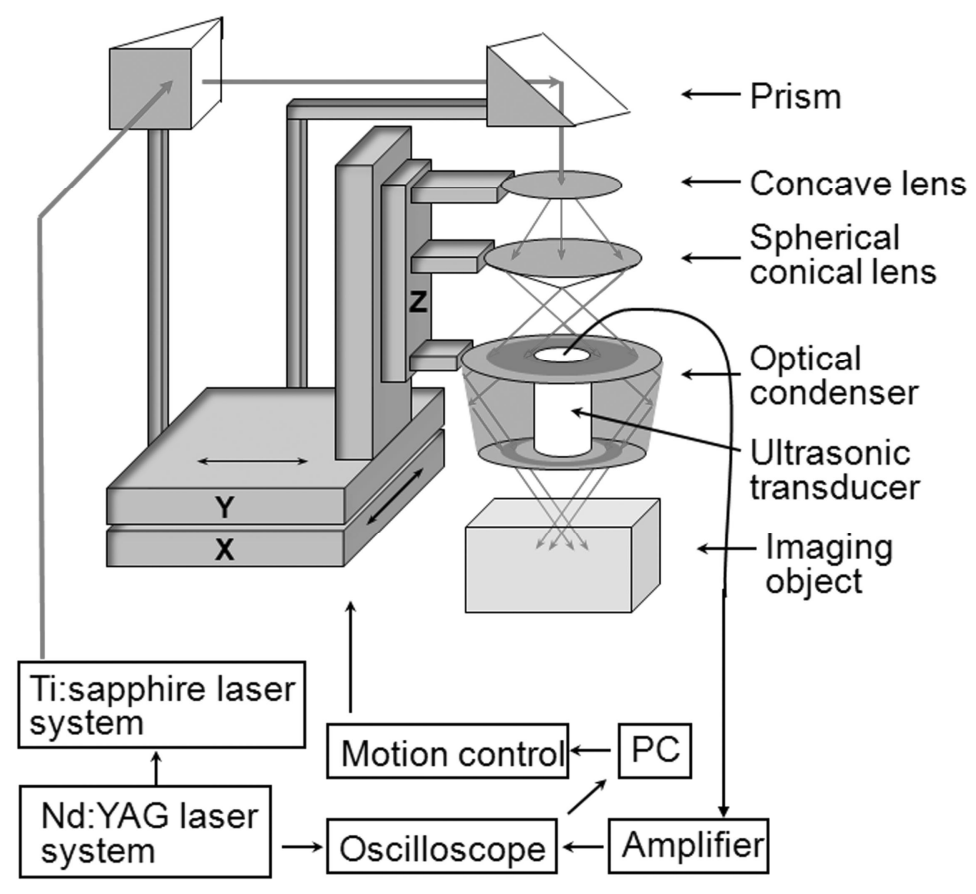

Figure 2. Reflection-mode photoacoustic imaging system for deeply located biological tissue imaging. See reference 30 for the detailed description. Concave lens, spherical conical lens, and optical condenser form dark ring-shape illumination, which can reduce surface interference.

Phantoms. To estimate the concentration of Au nanocages accumulated in a SLN of a rat ex vivo, phantom experiments were performed. Phantoms were made of $12 \%$ porcine gelatin containing Au nanocages of various concentrations (0.1-8 $\mathrm{nM}$ ). The phantoms (a cylinder with $3 \mathrm{~mm}$ in diameter and $4 \mathrm{~mm}$ in length) were made similarly in shape and size to a real SLN of a rat to rule out the effect of the size differences for PA signal measurement. PA signals were directly measured from these phantoms (Figure. 3B). Similarly, PA signals from excised real SLNs were also measured in the same way and compared with those from the phantoms to estimate the concentrations of Au nanocages accumulated in SLNs.

Animals. Sprague Dawley rats weighing 250-390 g were used. The rats were initially anesthetized with a mixture of Ketamine $(85 \mathrm{mg} / \mathrm{kg})$ and Xylazine $(15 \mathrm{mg} / \mathrm{kg})$. The region of interest was gently depilated using a commercial hairremoval lotion before imaging. An intradermal injection of $100 \mu \mathrm{L}$ of $2 \mathrm{nM}$ Au nanocage solution was performed on a left forepaw pad. During all image acquisitions, anesthesia was maintained using vaporized isoflurane $(1 \mathrm{~L} / \mathrm{min}$ oxygen and $0.75 \%$ isoflurane, Euthanex Corp.), and vitals were monitored using a pulseoximeter (NONIN Medical INC., $8600 \mathrm{~V})$. During the image acquisition, $8 \mathrm{~mL}$ of $0.9 \%$ saline was administered for hydration. After the image acquisition, the animals were euthanized by pentobarbital overdose. All animal experiments were carried out in compliance with the guidelines on the care and use of laboratory animals at Washington University in St. Louis. 


\section{EXPERIMENTAL RESULTS}

In a rat, an in vivo SLN containing Au nanocages was noninvasively imaged using the reflection-mode photoacoustic imaging system. Figure 2A shows a photograph of the axillae of the rat with hair removed before PA imaging. Figure 2B is a photograph of the same region with skin and fatty tissue removed, exposing the SLN containing Au nanocages after all PA images had been captured. The size of the oval SLN was $4 \mathrm{~mm}$ x $6 \mathrm{~mm}$. Before the injection of Au nanocages in the forepaw pad, a PA image was acquired as a control (Figure 2C). The vasculature within $\sim 3 \mathrm{~mm}$ below the skin surface was displayed in the form of a maximum amplitude projection (MAP).(31) For visibility, PA signals beyond the $3 \mathrm{~mm}$ depth were excluded in the image formation. After the injection of Au nanocages $(2 \mathrm{nM}$ and $0.1 \mathrm{~mL})$, scanning started immediately to evaluate the migration and accumulation time of the Au nanocages. PA signals from the SLN were detectable at about 13 min after the injection (Figure 2D). Here, the SLN was imaged with low contrast, implying low accumulation of Au nanocages at this time. Figures 2E-2G show PA saggital MAP images acquired at 73 min, 127 min, and 186 min after the injection, respectively. As time went by, the contrast at the SLN gradually increased, which means that more Au nanocages were gradually accumulated by the SLN.

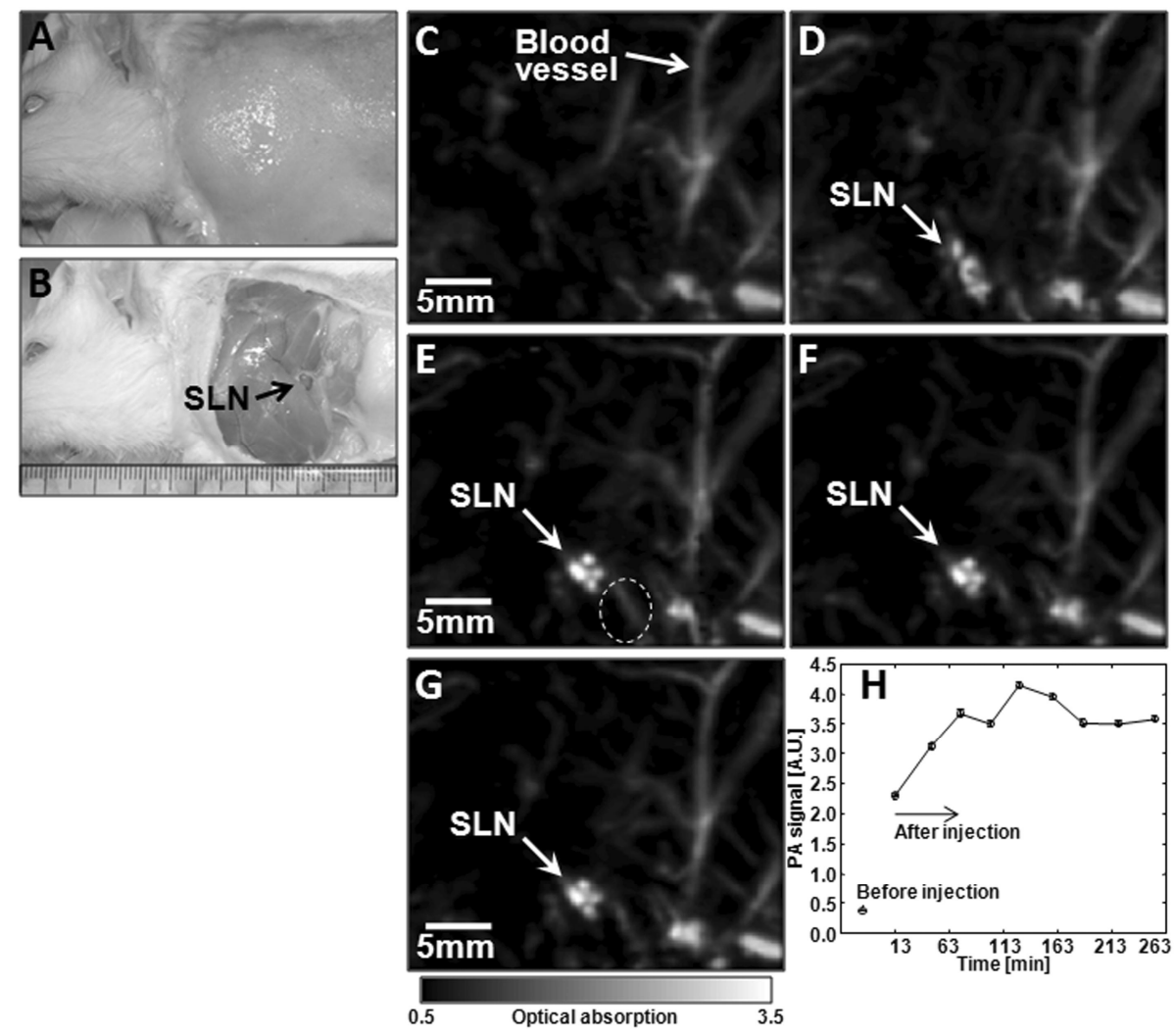

Figure 2. In vivo non-invasive photoacoustic time-course saggital MAP images. (A) Photograph of axillary region with hair removed. (B) Photograph with skin and fatty tissue removed after the photoacoustic images had been recorded. The dark blue dyed lymphatic channel on the bottom of the SLN is shown in the inset. Photoacoustic images acquired before (C) and after (D-G) the nanocage injection: (D) $13 \mathrm{~min}$ (SLN started to appear), (E) $73 \mathrm{~min}$, (F) $127 \mathrm{~min}$, (G) $186 \mathrm{~min}$. All images were acquired without signal averaging. $(\mathrm{H})$ Accumulations of nanocages in a SLN over time, in terms of the amplitude changes of PA signals. After the injection, PA signals increased with time, which means gradual accumulations of nanocages. Peak accumulation occurred at $140 \mathrm{~min}$ after the injection. PA signals from the SLN were normalized by those from adjacent blood vessels (the dotted box in Figure 2E) to minimize the ultrasonic focal effect, and normalized by maximum. Errorbar is standard error. SLN, sentinel lymph node. Colorbar represents optical absorptions. 
To evaluate time-dependent changes in Au nanocage accumulation, the averaged values of the PA signal amplitudes within the SLN were calculated. To minimize the off-focus effect of an ultrasonic transducer in the PA signal measurement, the calculated PA values were normalized by those from the adjacent blood vessel, indicated by the dotted circle in Figure 2E. Figure 2H shows the variation of accumulation in terms of PA signal amplitude versus the time after injection. The peak accumulation time of Au nanocages in the SLN was observed to be about 120 min after the injection. To estimate the concentration of Au nanocages accumulated in a SLN ex vivo, phantom experiments were conducted. The concept of this estimation is to compare PA signals from known-concentration phantoms with those from real SLNs. PA signals were obtained from the. To reduce the propagation error of ultrasonic waves caused by different shapes, phantoms were slightly squeezed and flattened. The measured PA signals were plotted in Figure 3A as a function of concentrations.

Eight SLNs from four rats were harvested at about $150 \mathrm{~min}$ after the injection to estimate the concentrations of $\mathrm{Au}$ nanocages in real SLNs. Figure 3B is the PA signal from eight SLNs. The SLNs was classified into two group, more accumulated and less accumulated one. In the inset of Figure 3B, ex vivo PA MAP images (1: more accumulated and 2: less accumulated) of two out of eight SLNs are shown as the examples. We measured PA signals from the eight real SLNs and derived the corresponding concentrations from Figure 3A. Five out of eight SLNs generated $0.2 \pm 0.02 \mathrm{mV}$ PA signals (standard deviation), which corresponds to a concentration of $6.9 \pm 0.29 \mathrm{nM}$ (standard deviation), higher than the concentration of the original solution by a factor of 3.5. As observed in Figures 3B, 1 and 2, the Au nanocages seemed to accumulate mostly in the subcapsular sinus of the node, and not drain much to the trabecular sinus. The function of the lymph node as a filter would explain this well. The rest of the SLNs produced PA signals of $0.02 \pm 0.003 \mathrm{mV}$ (standard deviation), which corresponds to a concentration of $0.15 \pm 0.016 \mathrm{nM}$ (standard deviation). The large variations in concentration of Au nanocage in the SLNs were thought to be related to the physiology of lymphatic systems, such as the rate of lymphatic flow,(32) intrinsic smooth muscle action,(33) and physical skeletal muscle movement.(34)
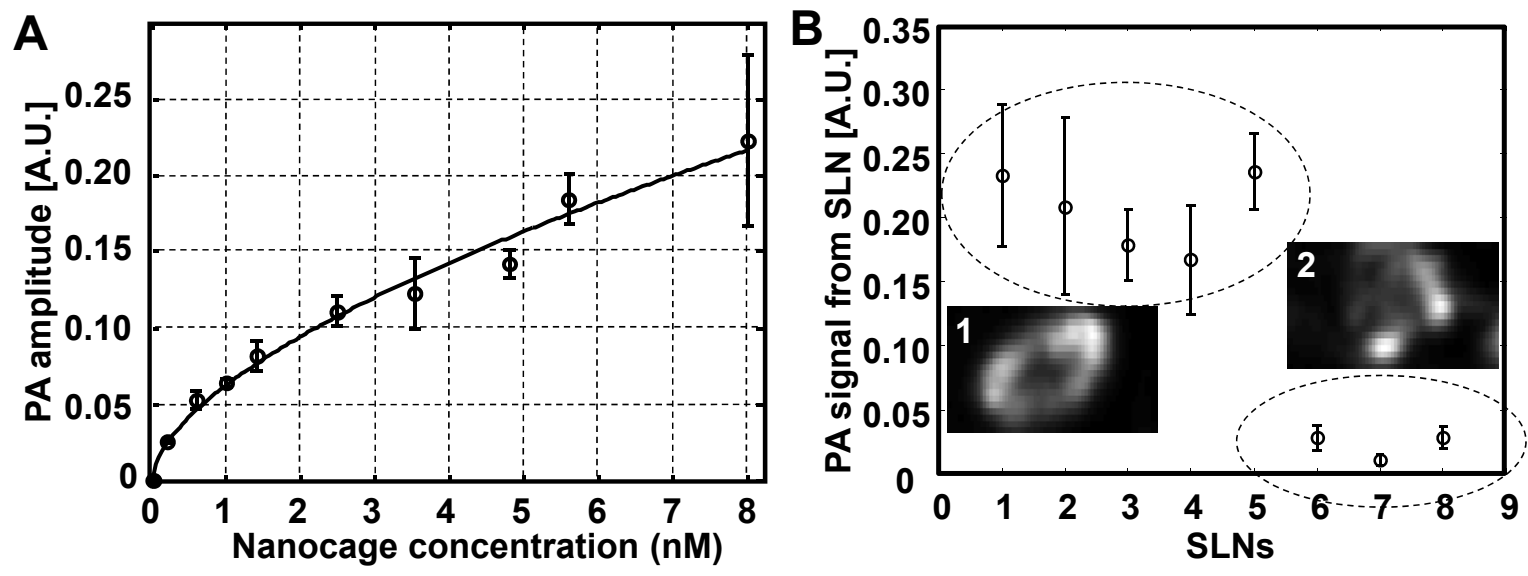

Figure 4. (A) PA signal from the gelatin phantom containing nanocages with various concentrations $(0-8 \mathrm{nM})$. All measured values were scaled down to $0 \mathrm{~dB}$ gain. Errorbar indicates standard deviation. (B) PA signals measured from eight real SLNs. Insets 1 and 2 show the PA MAP images representing more accumulated and less accumulated one, respectively. SLN, sentinel lymph node.

\section{DISCUSSION}

For future studies, several improvements concerning Au nanocages and the imaging system are recommended. First, an ideal mapping agent should be small enough to rapidly drain to lymphatic vessels and flow into lymph nodes, but large enough to stay within the lymphatic system during the procedure. Particles smaller than $5 \mathrm{~nm}$ can leak into capillary blood vessels and flow into the cardiovascular circulatory system. $(35,36)$ Once small particles $(5-10 \mathrm{~nm})$ drain 
to SLNs, they may quickly migrate to echelon lymph nodes; hence, they will increase the false positive rate. On the other hand, particles larger than $1000 \mathrm{~nm}$ mostly stay in the extracellular space (injection site). The Au nanocages that we used for this study had the average edge length of $50 \mathrm{~nm}$, and the peak accumulation time was observed to be about $140 \mathrm{~min}$. Even though the relationship between size and migration time for a certain shape was reported,(14) this will depend upon the shapes as well as the sizes of the tracers. Therefore, it is necessary to find an optimal size of Au nanocages for SLN mapping to secure fast migration, sufficient duration of trapping for imaging, and sufficiently strong PA signal generation for imaging deep sites. Secondly, even though various kinds of nanoparticles have widely been studied for biomedical applications, their potential toxicity currently limits their clinical use. For this study, we were not able to investigate the distribution of Au nanocages in a rat due to technical limitations. Unlike quantum dots,(21) however, Aubased nanoparticles do not have problems with heavy metal toxicity. Moreover, gold has been approved and used for human treatment. $(21,37)$

We observed the higher concentration than the original one of Au nanocages accumulated in the subcapsular sinus of a SLN, but did not observe a solid trend of their clearance in the duration of measurement (up to 5 hours). For SLN mapping, the higher accumulation would be beneficial, allowing a greater imaging depth with sufficient SNR. However, the higher accumulation and prolonged stay of Au nanocages may increase the chance of potential toxicity. Size adjustment and PEGylation of Au nanocages might reduce this prolonged stay,(38) so Au nanocages may be more efficiently excreted from the kidney.(14)

The last concern is that since the PA imaging system for this study employed a spherically focused ultrasonic transducer and a $10 \mathrm{~Hz}$ pulse-repetition-rate laser system, the scanning time for a 3D image was $\sim 25$ min with $0.2 \mathrm{~mm}$ and $0.35 \mathrm{~mm}$ stepsizes. In addition, the system was designed to sit on a table. The slow imaging speed and the tabletop design may not allow the imaging system to be directly applied in clinic. Employing an ultrasonic array system and a higher pulse-repetition-frequency laser system can dramatically improve the scanning time and flexibility of the scanner.

\section{CONCLUSION}

We successfully accomplished noninvasive in vivo Au nanocage-contrast-enhanced SLN identification using PA imaging modality in a rat model. Compared to the SLN mapping methods stated previously, the Au nanocage based PA method has several advantages: 1) noninvasive mapping, 2) peak optical absorption at the near-infrared region, allowing deeper imaging penetration than at the visible range, 3) rapid drainage into lymphatic channels, 4) accumulation of Au nanocages in excess of the original concentration, generating stronger PA signals (high SNR), 5) good spatial resolution, and 6) low cost. To evaluate this technique for clinical use, we also demonstrated that the proposed method could reach as deep as $33 \mathrm{~mm}$ below the skin surface with good contrast. This depth is greater than the mean depth of SLNs in human beings. Once Au nanocages are approved by the FDA, the next step will be a pilot study on human beings. In addition, with the aid of bioconjugation with antibodies for specific cancer cells, the Au nanocage based method can potentially provide noninvasive SLNB, while allowing simultaneous SLN identification.

\section{ACKNOWLEDGEMENT}

This research was sponsored in part by grants from National Institutes of Health (R01 EB000712 and R01 NS46214 to L.V.W.). Y.X. thanks the National Institutes of Health for a 2006 Director's Pioneer Award (5DP1OD000798-04).

\section{REFERENCE}

1. S. K. Sahoo and V. Labhasetwar, "Nanotech approaches to drug delivery and imaging," Drug discovery today 8(24), 1112-1120 (2003)

2. J. Panyam and V. Labhasetwar, "Biodegradable nanoparticles for drug and gene delivery to cells and tissue," Advanced drug delivery reviews 55(3), 329-347 (2003) 
3. L. D. True and X. Gao, "Quantum dots for molecular pathology: their time has arrived," J Mol Diagn 9(1), 7-11 (2007)

4. X. Gao, Y. Cui, R. M. Levenson, L. W. Chung and S. Nie, "In vivo cancer targeting and imaging with semiconductor quantum dots," Nature biotechnology 22(8), 969-976 (2004)

5. P. M. Winter, S. D. Caruthers, A. Kassner, T. D. Harris, L. K. Chinen, J. S. Allen, E. K. Lacy, H. Zhang, J. D. Robertson, S. A. Wickline and G. M. Lanza, "Molecular imaging of angiogenesis in nascent Vx-2 rabbit tumors using a novel alpha(nu)beta3-targeted nanoparticle and 1.5 tesla magnetic resonance imaging," Cancer research 63(18), 5838-5843 (2003)

6. M. G. Harisinghani, J. Barentsz, P. F. Hahn, W. M. Deserno, S. Tabatabaei, C. H. van de Kaa, J. de la Rosette and R. Weissleder, "Noninvasive detection of clinically occult lymph-node metastases in prostate cancer," The New England journal of medicine 348(25), 2491-2499 (2003)

7. S. Kim, Y. T. Lim, E. G. Soltesz, A. M. De Grand, J. Lee, A. Nakayama, J. A. Parker, T. Mihaljevic, R. G. Laurence, D. M. Dor, L. H. Cohn, M. G. Bawendi and J. V. Frangioni, "Near-infrared fluorescent type II quantum dots for sentinel lymph node mapping," Nature biotechnology 22(1), 93-97 (2004)

8. Y. W. Wang, X. Y. Xie, X. D. Wang, G. Ku, K. L. Gill, D. P. O'Neal, G. Stoica and L. V. Wang, "Photoacoustic tomography of a nanoshell contrast agent in the in vivo rat brain," Nano letters 4(9), 1689-1692 (2004)

9. H. Kobayashi and M. W. Brechbiel, "Dendrimer-based nanosized MRI contrast agents," Curr Pharm Biotechnol 5(6), 539-549 (2004)

10. O. Rabin, J. Manuel Perez, J. Grimm, G. Wojtkiewicz and R. Weissleder, "An X-ray computed tomography imaging agent based on long-circulating bismuth sulphide nanoparticles," Nat Mater 5(2), 118-122 (2006)

11. D. L. Morton, D. R. Wen, J. H. Wong, J. S. Economou, L. A. Cagle, F. K. Storm, L. J. Foshag and A. J. Cochran, "Technical details of intraoperative lymphatic mapping for early stage melanoma," Arch Surg 127(4), 392-399 (1992)

12. D. N. Krag, D. L. Weaver, J. C. Alex and J. T. Fairbank, "Surgical resection and radiolocalization of the sentinel lymph node in breast cancer using a gamma probe," Surgical oncology 2(6), 335-339; discussion 340 (1993)

13. Y. Koyama, V. S. Talanov, M. Bernardo, Y. Hama, C. A. Regino, M. W. Brechbiel, P. L. Choyke and H. Kobayashi, "A dendrimer-based nanosized contrast agent dual-labeled for magnetic resonance and optical fluorescence imaging to localize the sentinel lymph node in mice," J Magn Reson Imaging 25(4), 866-871 (2007)

14. M. Nakajima, M. Takeda, M. Kobayashi, S. Suzuki and N. Ohuchi, "Nano-sized fluorescent particles as new tracers for sentinel node detection: Experimental model for decision of appropriate size and wavelength," Cancer Science 96(6), 353-356 (2005)

15. R. H. Oyen, H. P. Van Poppel, F. E. Ameye, W. A. Van de Voorde, A. L. Baert and L. V. Baert, "Lymph node staging of localized prostatic carcinoma with CT and CT-guided fine-needle aspiration biopsy: prospective study of 285 patients," Radiology 190(2), 315-322 (1994)

16. S. Saini, R. Sharma, R. L. Baron, D. A. Turner, P. R. Ros, P. F. Hahn, W. C. Small, E. E. Delange, A. E. Stillman, R. R. Edelman, V. M. Runge and E. K. Outwater, "Multicentre dose-ranging study on the efficacy of USPIO ferumoxtran-10 for liver MR imaging," Clinical radiology 55(9), 690-695 (2000)

17. S. C. Michel, T. M. Keller, J. M. Frohlich, D. Fink, R. Caduff, B. Seifert, B. Marincek and R. A. Kubik-Huch, "Preoperative breast cancer staging: MR imaging of the axilla with ultrasmall superparamagnetic iron oxide enhancement," Radiology 225(2), 527-536 (2002)

18. M. G. Torchia, R. Nason, R. Danzinger, J. M. Lewis and J. A. Thliveris, "Interstitial MR lymphangiography for the detection of sentinel lymph nodes," Journal of surgical oncology 78(3), 151-156; discussion 157 (2001)

19. C. P. Parungo, Y. L. Colson, S. W. Kim, S. Kim, L. H. Cohn, M. G. Bawendi and J. V. Frangioni, "Sentinel lymph node mapping of the pleural space," Chest 127(5), 1799-1804 (2005)

20. J. A. Margenthaler, "Depth measurement of sentinel lymph nodes in human," (2007).

21. A. G. Cuenca, H. Jiang, S. N. Hochwald, M. Delano, W. G. Cance and S. R. Grobmyer, "Emerging implications of nanotechnology on cancer diagnostics and therapeutics," Cancer 107(3), 459-466 (2006)

22. K. H. Song, E. W. Stein, J. A. Margenthaler and L. V. Wang, "Noninvasive photoacoustic identification of sentinel lymph nodes containing methylene blue in vivo in a rat model," Journal of biomedical optics 08070R (In press $)((2008)$

23. G. Ku and L. V. Wang, "Deeply penetrating photoacoustic tomography in biological tissues enhanced with an optical contrast agent," Optics letters 30(5), 507-509 (2005)

24. S. Sohrabnezhad, A. Pourahmad and M. A. Sadjadi, "New methylene blue incorporated in mordenite zeolite as humidity sensor material," Materials Letters 61(11-12), 2311-2314 (2007) 
25. S. E. Skrabalak, J. Chen, L. Au, X. Lu, X. Li and Y. Xia, "Gold nanocages for biomedical applications," Adv Mater 19(20), 3177-3184 (2007)

26. J. Chen, F. Saeki, B. J. Wiley, H. Cang, M. J. Cobb, Z. Y. Li, L. Au, H. Zhang, M. B. Kimmey, X. Li and Y. Xia, "Gold nanocages: bioconjugation and their potential use as optical imaging contrast agents," Nano letters 5(3), 473477 (2005)

27. M. Hu, J. Y. Chen, Z. Y. Li, L. Au, G. V. Hartland, X. D. Li, M. Marquez and Y. N. Xia, "Gold nanostructures: engineering their plasmonic properties for biomedical applications," Chem Soc Rev 35(11), 1084-1094 (2006)

28. Y. Sun and Y. Xia, "Mechanistic study on the replacement reaction between silver nanostructures and chloroauric acid in aqueous medium," Journal of the American Chemical Society 126(12), 3892-3901 (2004)

29. S. E. Skrabalak, L. Au, X. D. Li and Y. Xia, "Facile synthesis of Ag nanocubes and Au nanocages," Nat Protoc 2(9), 2182-2190 (2007)

30. American, National, Standards and Institute, "American National Standard for the Safe Use of Lasers ANSI Z136.1-2000," (New York, 2000).

31. K. H. Song and L. V. Wang, "Deep reflection-mode photoacoustic imaging of biological tissue," Journal of biomedical optics 12(6), 060503 (2007)

32. R. F. Uren, R. B. Howman-Giles and J. F. Thompson, "Demonstration of second-tier lymph nodes during preoperative lymphoscintigraphy for melanoma: incidence varies with primary tumor site," Ann Surg Oncol 5(6), 517-521 (1998)

33. S. M. Moghimi and B. Bonnemain, "Subcutaneous and intravenous delivery of diagnostic agents to the lymphatic system: applications in lymphoscintigraphy and indirect lymphography," Advanced drug delivery reviews 37(1-3), 295-312 (1999)

34. F. Ikomi, J. Hunt, G. Hanna and G. W. Schmid-Schonbein, "Interstitial fluid, plasma protein, colloid, and leukocyte uptake into initial lymphatics," J Appl Physiol 81(5), 2060-2067 (1996)

35. N. P. Alazraki, D. Eshima, L. A. Eshima, S. C. Herda, D. R. Murray, J. P. Vansant and A. T. Taylor, "Lymphoscintigraphy, the sentinel node concept, and the intraoperative gamma probe in melanoma, breast cancer, and other potential cancers," Seminars in nuclear medicine 27(1), 55-67 (1997)

36. E. G. Soltesz, S. Kim, R. G. Laurence, A. M. DeGrand, C. P. Parungo, D. M. Dor, L. H. Cohn, M. G. Bawendi, J. V. Frangioni and T. Mihaljevic, "Intraoperative sentinel lymph node mapping of the lung using near-infrared fluorescent quantum dots," The Annals of thoracic surgery 79(1), 269-277; discussion 269-277 (2005)

37. P. L. Mottram, "Past, present and future drug treatment for rheumatoid arthritis and systemic lupus erythematosus," Immunology and cell biology 81(5), 350-353 (2003)

38. H. Otsuka, Y. Nagasaki and K. Kataoka, "PEGylated nanoparticles for biological and pharmaceutical applications," Advanced drug delivery reviews 55(3), 403-419 (2003) 